Japanese Journal of Physiology, 32, 807-816, 1982

\title{
Blood Flow Redistribution in the Transposition Response of the Rat
}

\author{
Juro IrIUChIJIMA, Yasushi Kawaue, and Yasuhiro Teranishi \\ Department of Physiology, School of Medicine, University of \\ Hiroshima, Hiroshima, 734 Japan
}

\begin{abstract}
Changes in blood flow in different arteries occurring in the transposition response of the rat, induced by transposing the animal from a "home" cage to a new one, were studied using chronically implanted electromagnetic flow probes. In the response, hindquarter flow was increased by more than $50 \%$, superior mesenteric and renal flows were decreased by about 30 and $15 \%$, respectively, and common carotid flow remained almost unchanged. The increase in hindquarter flow in the response was much less after $\beta$-adrenoceptor blockade with propranolol. In adrenalectomized rats the flow changes in the hindquarter, mesenteric, and renal areas were less marked in either direction. These findings indicate that the blood flow redistribution in the transposition response is characterized by a shift of flow from the viscera to the skeletal muscles, in which the $\alpha$ - and $\beta$-effects of catecholamines released from the adrenal medulla in the response play a dominant role. Key Words: transposition response, flow distribution, adrenal medulla.
\end{abstract}

The transposition response is a cardiovascular response complex which is observed when a rat is transposed from its usual cage to a new one, and is considered to be triggered by the stress of change of habitat and effected by an excitation of the sympathoadrenal system (IrIUCHIJIMA et al., 1980). Although arterial pressure remains almost unchanged in the response, heart rate and minute volume are increased considerably and total peripheral resistance is decreased. Such a response may be designated an isopressor response. After $\beta$-adrenoceptor blockade with propranolol, the response is converted to a pressor one in which arterial pressure and total peripheral resistance are increased. These findings indicate that $\beta$-adrenergic vasodilatation is one of the major components of the transposition response, besides an excitation of the cardiac sympathetic nerves and $\alpha$-adrenergic vasoconstrictor mechanisms.

The purpose of this study is to find the peripheral vascular area which is responsible for the marked vasodilatation occurring in the transposition response. An electromagnetic flow probe was chronically implanted at the common carotid

Received for publication April 5, 1982

入内島十郎，川上恭司，寺西泰弘 
artery, superior mesenteric artery, renal artery, or terminal aorta in the rat to observe the change in flow at these arteries in the transposition response. It was found that the hindquarter flow measured at the terminal aorta was markedly increased during the response, which was for the most part ascribable to the $\beta$ effect of circulatory catecholamines released from the adrenal medulla.

\section{METHODS}

Peripheral flow measurement in conscious rats. Male 10-20 week-old Wistar rats were anesthetized by an intraperitoneal injection of $50 \mathrm{mg} / \mathrm{kg}$ of thiamylal sodium. A Nihon Kohden electromagnetic flow probe was implanted around the left common carotid artery (diameter of probe: 1 or $1.5 \mathrm{~mm}$ ), superior mesenteric artery $(1$ or $1.5 \mathrm{~mm})$, left renal artery $(0.8$ or $1 \mathrm{~mm})$, or terminal aorta $(1.5,2$, or $2.5 \mathrm{~mm})$ above the iliac bifurcation. The common carotid artery was reached by a median incision in the ventral neck, and the latter three arteries, retroperitoneally by a flank incision. The lead wires from the probe were passed under the skin, led to the dorsal neck and there exteriorized. Flow recording was performed by connecting the plug at the end of the wires to the cable from the flowmeter circuit (Nihon Kohden MFV-1100). Flow probes were calibrated before implantation by passing a known amount of $0.9 \%$ saline through excised arteries. After implantation of a probe around an artery the point of zero flow was defined either by stopping the heart for a while reflexively by digital compression of the bilateral eyeballs (for terminal aorta and carotid artery) or by momentarily occluding the artery by digital compression directly applied to the artery through the skin (for superior mesenteric, renal and carotid arteries). Flow signal was smoothed out with a CR integrating circuit with a time constant of 0.1 or $0.3 \mathrm{sec}$ to obtain mean flow. In most experiments instantaneous flow was also recorded in parallel with the mean flow to check occasional artifacts. Flow was normalized to $100 \mathrm{~g}$ of body weight.

Induction of transposition response. Rats implanted with a flow probe were housed individually in white polyethylene cages measuring approximately $35 \times$ $30 \times 17$ (depth) $\mathrm{cm}$. Water and pellets were given ad libitum. The floor of a cage was covered with wood chips. Usually, flow recording was started 3 days after operation and most recording was done in the morning. The transposition response was induced by the following procedure: After observing flow in a rat in the "home" cage when the rat was apparently at rest, the animal was lifted by hand and placed in a new cage also made of white polyethylene but smaller in size $(c a .30 \times 20 \times 13 \mathrm{~cm})$ and without chips. After determining a relatively steady flow level in this new environment, usually in 30-60 sec, the rat was returned to the "home" cage again by hand.

Adrenalectomy. After normal flow measurements had been completed in a few days, adrenalectomy was performed in most rats under ether anesthesia. 
By median laparotomy, the bilateral adrenals-both medulla and cortex-were removed. After adrenalectomy, an adrenocortical hormone, dexamethasone sodium sulfate $(0.2 \mathrm{mg}$, s.c.), was injected daily. Most measurements in adrenalectomized rats were performed 2 or 3 days after adrenalectomy. In some rats arterial cannulation for pressure measurement was performed instead of or a few days before or after adrenalectomy. Arterial cannulation was not done in most rats because this involves the sacrifice of one femoral artery and interferes with the blood flow distribution.

\section{RESULTS}

Flow change in transposition response

Each respective example of the flow change occurring in the four arteries during the transposition response is presented in Fig. 1. In each record, at the time marked $T$, the rat which had been grasped by hand and lifted from the

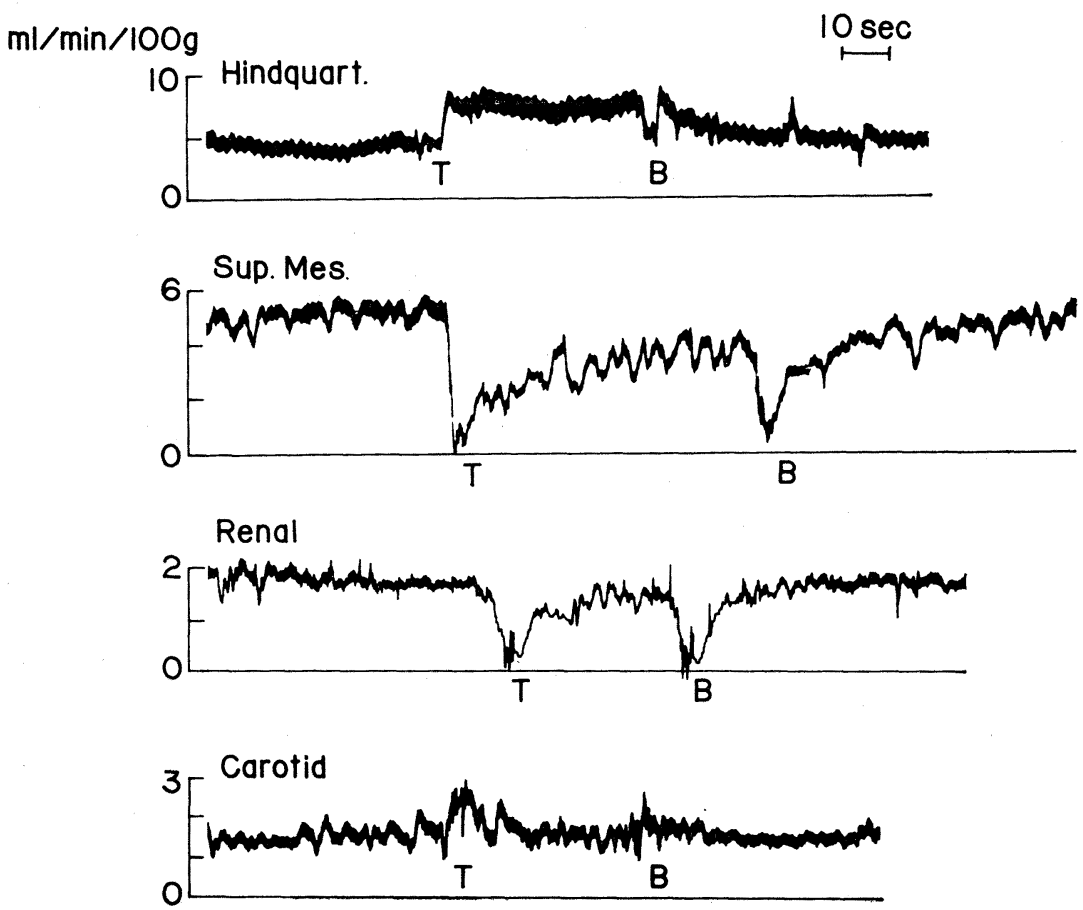

Fig. 1. Changes in blood flow at different arteries in conscious rats in the transposition response. In each record the rat, which had been grasped by hand and lifted from the "home" cage several seconds before, was released into a new cage at the time marked T. The relatively steady flow level reached while the rat was in the new cage was designated as that occurring in the transposition response. Subsequently the rat was grasped again to be returned to the home cage at $\mathrm{B}$.

Vol. 32, No. 5, 1982 

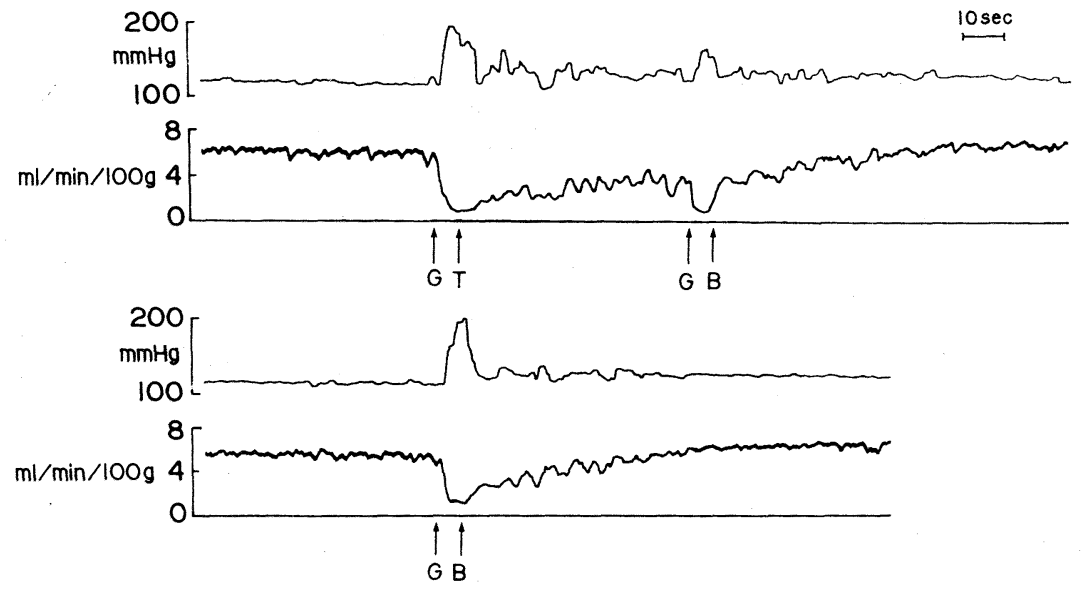

Fig. 2. Simultaneous recording of mean arterial pressure and superior mesenteric flow in a conscious rat. The rat was grasped by hand at the time marked $\mathrm{G}$ and released to the new cage at $T$. It was returned to the "home" cage at B. In the lower pair of the records, the rat was returned to the "home" cage after grasping only, instead of transposing it to the new cage as in the upper pair.

"home" cage was released into a new cage. The rat was then left in the new cage until a relatively steady new flow level was reached. This flow level was designated as that occurring during the transposition response. Thereafter, the rat was returned to the "home" cage at time B. While the rat was being held for transposition or for returning to the "home" cage, the flows in the superior mesenteric and renal arteries decreased markedly. Concomitantly the arterial pressure was elevated considerably (Fig. 2, upper pair). For convenience, this response complex occurring when a rat is being grasped is referred to as the "grasp response." That the transposition response is not an after-effect of the grasp response is to be seen from comparing the two records in Fig. 2. When a rat was released into the old "home" cage after grasping only (Fig. 2, lower pair), the superior mesenteric flow, without changing to a new level, returned to the previous level similarly as when the rat was returned from a new cage to the "home" cage (Fig. 2, upper pair, right). During the grasp response the hindquarter and carotid flows did not change appreciably in the face of the marked rise in arterial pressure. This suggests that sympathetic vasoconstrictor discharge to the areas supplied by these arteries was concomitantly increased.

The means with standard deviation from several rats for the flow levels in each artery before and during the transposition response are presented in Table 1 . In compiling these and the following summary data, unless otherwise noted, the flow value for each individual rat was the mean of all observations repeated during a day or over several consecutive days. The means and standard deviations in the table were calculated from the flow values representing individual rats. Thus, 
Table 1. Peripheral blood flow before and during the transposition response.

\begin{tabular}{lcccccc}
\hline & $n$ & $\begin{array}{c}\text { Before } \\
(\mathrm{ml} /(\mathrm{min} .\end{array}$ & $\begin{array}{c}\text { During } \\
(\mathrm{ml} /(\mathrm{min}) \\
100 \mathrm{~g})\end{array}$ & $\begin{array}{c}\text { Difference } \\
(\mathrm{ml} /(\mathrm{min} \cdot 100 \mathrm{~g}))\end{array}$ & $\%$ Change & $P<$ \\
\hline Hindq. & 9 & $5.81 \pm 1.19$ & $9.73 \pm 2.31$ & $3.89 \pm 1.53$ & $67.5 \pm 20.1$ & 0.001 \\
Sup. mes. & 6 & $4.57 \pm 0.638$ & $3.28 \pm 0.600$ & $-1.29 \pm 0.363$ & $-28.3 \pm 7.74$ & 0.001 \\
Renal & 7 & $1.77 \pm 0.333$ & $1.49 \pm 0.349$ & $-0.276 \pm 0.0919$ & $-16.1 \pm 6.02$ & 0.001 \\
Carot. & 6 & $1.64 \pm 0.208$ & $1.80 \pm 0.337$ & $0.163 \pm 0.195$ & $9.46 \pm 11.4$ & $\mathrm{NS}$ \\
$\mathrm{H}+\mathrm{S}+$ 2(R+C) & 17.2 & 19.6 & 2.37 & & \\
\hline
\end{tabular}

Values are mean \pm S.D. The figures on the bottom line represent the sum of the means of flow after doubling those of bilateral arteries.

the $n$ in the table represents the number of rats and not the number of observations.

In the transposition response the hindquarter flow was increased by more than $50 \%$. On the contrary the superior mesenteric flow was markedly decreased. The renal flow was also decreased significantly but less so than the superior mesenteric flow. The change in the carotid flow in the response varied greatly from rat to rat and also from trial to trial in the same rat. By and large it remained almost unchanged in the transposition response. Although not included in the table because of the small number of observations, the flow in the celiac artery was decreased during the transposition response as markedly as that in the superior mesenteric artery.

Usually, the hindquarter flow was increased when the rat moved using its hind limbs. As stated above, the transposition response was induced only when the rat was apparently at rest. After the transposition had been completed the rat frequently moved about in its new cage. However, the level of the increased hindquarter blood flow occurring during the response was similar no matter whether the rat moved or remained quiet.

To have a rough idea on the net flow balance, the mean flow values in Table 1 were added for the four arteries, after doubling those for the renal and carotid arteries because, there, flow measurement was performed only at one of the bilateral arteries. The sum was $17.2 \mathrm{ml} /(\mathrm{min} \cdot 100 \mathrm{~g})$ before the transposition response, increasing by 2.37 to 19.6 during the response. This is consistent with our previous observation that cardiac output is increased in the transposition response (IrIUCHIJIMA et al., 1980).

\section{Effect of $\beta$-adrenoceptor blockade}

Since $\beta$-blockade with propranolol abolishes the increase in cardiac output in the transposition response (IRIUCHIJIMA et al., 1980), this procedure was expected to have considerable influence on regional flows also. After propranolol, the hindquarter flow was still increased significantly in the transposition response but was much less in magnitude. One example of comparison of flow change in the transposition response before and $30 \mathrm{~min}$ after propranolol administration $(1 \mathrm{mg} / \mathrm{kg}$, i.p.) is presented in Fig. 3 (compare top and middle records). The 

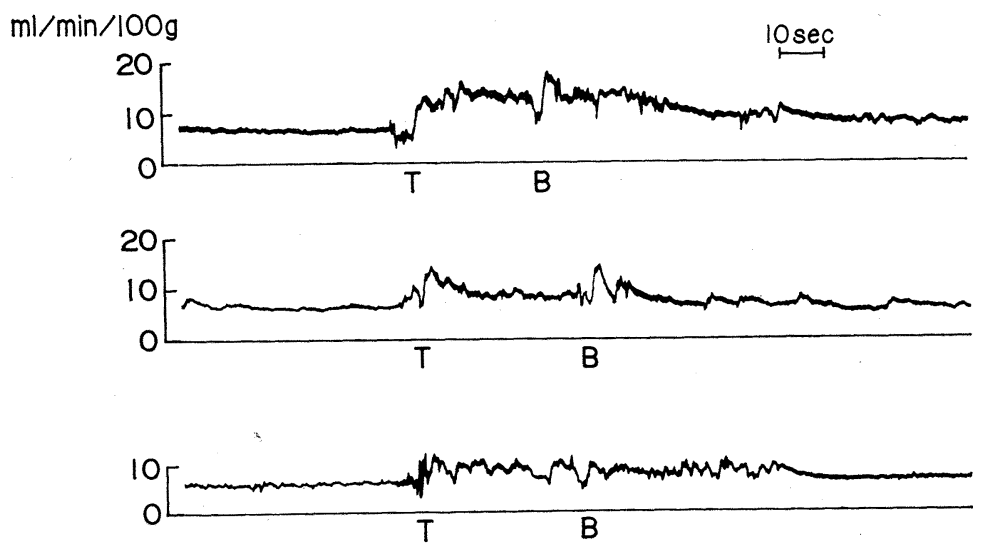

Fig. 3. Change in hindquarter flow in the transposition response. In each record the rat was transposed to the new cage at $\mathrm{T}$ and returned to the "home" cage at $\mathrm{B}$. Top: control. Middle: $30 \mathrm{~min}$ after propranolol $(1 \mathrm{mg} / \mathrm{kg}$, i.p.). Bottom: after adrenalectomy. The flow increase in the response was greatly diminished after either propranolol or adrenalectomy.

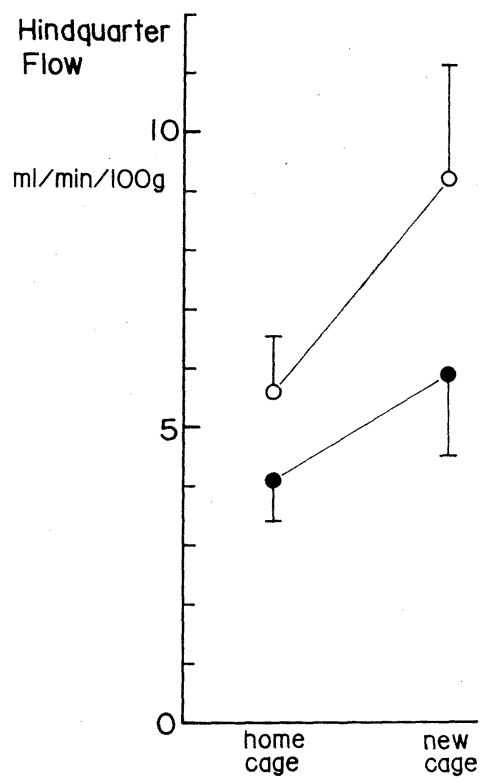

Fig. 4. Hindquarter flow before (home cage) and during the transposition response (new cage). Mean \pm S.D., $n=7$. Open circles: controls. Filled circles: $30 \mathrm{~min}$ after propranolol $1 \mathrm{mg} / \mathrm{kg}$, i.p. 
summary data from 7 rats are graphically displayed in Fig. 4. By $\beta$-adrenoceptor blockade, the femoral flow at rest decreased significantly $(-1.52 \pm 0.731 \mathrm{ml} /$ $(\min \cdot 100 \mathrm{~g}$ ), mean \pm S.D., $n=7, P<0.005)$. After $\beta$-adrenoceptor blockade the hindquarter flow was increased in the transposition response by $+1.78 \pm 0.877 \mathrm{ml} /$ $(\min \cdot 100 \mathrm{~g})$ or $+42.7 \pm 18.5 \%, P<0.005$. The increment was significantly less than that before blockade $(P<0.005)$.

In the other 3 arteries the $\beta$-adrenoceptor blockade with propranolol had no marked effect on either the flow at rest or the flow change in the transposition response. However, the decrease in superior mesenteric flow in the transposition response was less after blockade than before $(-22.7 \pm 11.6 \%$ vs. $-38.0 \pm$ $12.5 \%, n=5, P<0.05)$. The corresponding figures for the renal artery were $-21.0 \pm 4.23 \%$ vs. $-19.7 \pm 13.7 \%, n=4$, and those for the carotid artery were $-0.724 \pm 8.00 \%$ vs. $+3.09 \pm 7.17 \%, n=5$.

\section{Effect of adrenalectomy}

The hindquarter flow was still increased after adrenalectomy in the transposition response but much less in extent than before adrenalectomy (Fig. 3 bottom, compare with top). The mean hindquarter flow from 4 rats increased from $4.69 \pm$ 1.74 to $6.66 \pm 2.50 \mathrm{ml} /(\mathrm{min} \cdot 100 \mathrm{~g}),+41.4 \pm 16.1 \%, P<0.025$. This hindquarter flow increase during the transposition response in adrenalectomized rats was almost unaffected by propranolol ( $1 \mathrm{mg} / \mathrm{kg}$, i.p., after $30 \mathrm{~min})$ (Fig. 5), the flow change being $5.04 \pm 2.28$ to $6.73 \pm 2.43 \mathrm{ml} /(\mathrm{min} \cdot 100 \mathrm{~g}), n=4,+39.5 \pm 30.3 \%, P<0.10$.

After adrenalectomy the decrease in superior mesenteric flow in the transposition response was also less marked than before (Fig. 6). However, the decrease in superior mesenteric and renal flows in the grasp response, preceding the transposition response, was almost as marked as before adrenalectomy. In adrenalectomized rats during the transposition response, the superior mesenteric flow
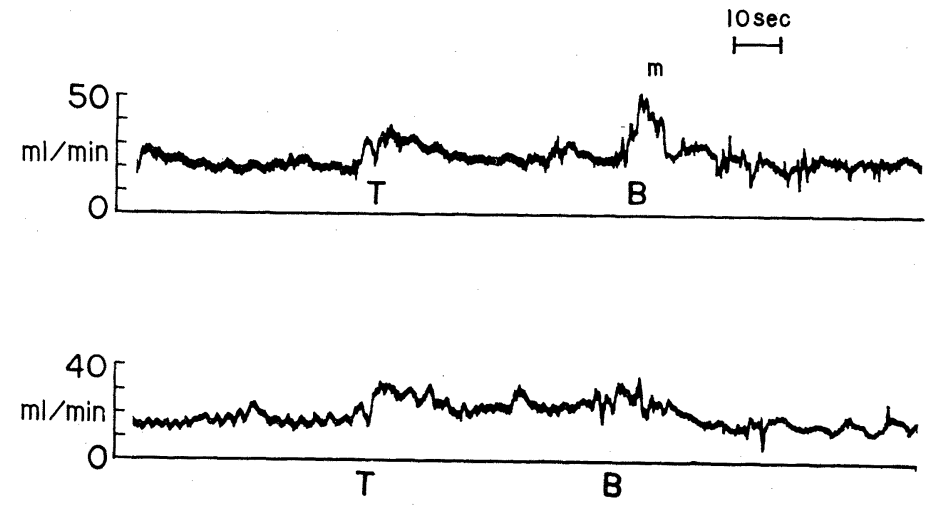

Fig. 5. Hindquarter flow in an adrenalectomized rat before (top) and after (bottom) propranolol $(1 \mathrm{mg} / \mathrm{kg}$, i.p.). The rat was transposed at $\mathrm{T}$ and returned at $\mathrm{B}$. $\mathrm{m}$ indicates that the increase in flow near this mark was induced by voluntary movement. 


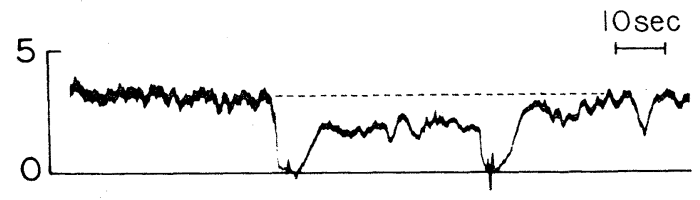

$\mathrm{mi} / \mathrm{min} / \mathrm{lOOg}$

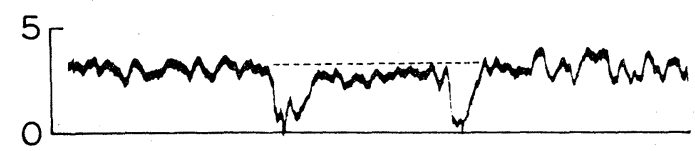

Fig. 6. Change in superior mesenteric flow in the transposition response before (top) and after (bottom) adrenalectomy. The decrease in flow in the response was less in adrenalectomized rats. The dotted line indicates the control flow level.

was decreased from $3.72 \pm 0.620$ to $3.18 \pm 0.891 \mathrm{ml} /(\mathrm{min} \cdot 100 \mathrm{~g}), n=6,-16.1 \pm$ $10.6 \%, P<0.025$. Before adrenalectomy the flow change in the same group of 6 rats was from $3.99 \pm 0.827 \mathrm{ml} /(\mathrm{min} \cdot 100 \mathrm{~g})$ to $2.77 \pm 0.840,-30.7 \pm 13.0 \%, P<$ 0.005 . These two changes were significantly different at $P<0.025$.

After adrenalectomy, the renal flow decrease in the transposition response was also less than before. The mean decrease values from 19 trials in 3 rats were: from $1.61 \pm 0.174 \mathrm{ml} /(\mathrm{min} \cdot 100 \mathrm{~g})$ to $1.48 \pm 0.173,-8.01 \pm 9.33 \%, P<0.005(n=$ 19). On the contrary, the carotid flow increased from $1.18 \pm 0.263$ to $1.40 \pm 0.351$, $+18.5 \pm 12.7 \%, P<0.001$ in $16(=n)$ trials in 3 rats.

\section{DISCUSSION}

During the transposition response of the rat in which cardiac output was increased by about $20 \%$, from about 20 to $24 \mathrm{ml} /(\mathrm{min} \cdot 100 \mathrm{~g}$ ) (IRIUCHIJMA et al., 1980), peripheral blood flow was observed to increase only at the terminal aorta. The increase in the hindquarter flow, which was about $4 \mathrm{ml} /(\mathrm{min} \cdot 100 \mathrm{~g})$ (Table 1), was just enough to account for the increase in the cardiac output. However, since superior mesenteric and renal flow as well as celiac flow were decreased in the response, an increase in flow comparable in absolute magnitude to the flow decrease in the viscera must occur in areas other than those whose flow was measured in the present study. The forelimbs and carcass are the candidates for such areas.

Considering the marked susceptibility of the flow increase in the hindquarters in the transposition response to adrenoceptor blockade, the vascular bed where flow is markedly increased in the response is considered to be that in skeletal muscle (GREEN and KEPCHAR, 1959). Taking into consideration that the increase in hindquarter flow in the transposition response was greatly diminished in adrenalectomized rats, the increase in hindquarter flow and cardiac output in general in the response is largely ascribable to a vasodilatation in skeletal muscle due to catecholamines released from the adrenal medulla. 
Administration of propranolol decreases the resting level of cardiac output (IRIUCHIJIMA et al., 1980). In the present study it was found that this procedure significantly decreased the resting hindquarter flow level. These findings suggest that, in the rat, the muscle vascular bed is maintained in a dilated state by a constant release of catecholamines from the adrenal medulla even when the rat is at rest. The major part of the catecholamines is considered to consist of adrenaline (SHePherd and West, 1951; Green and Miller, 1966).

The slight increase in hindquarter flow in the transposition response after $\beta$-adrenoceptor blockade may be partly ascribable to the increase in arterial pressure, since the blockade converts the transposition response from an isopressor to a pressor response (IRIUCHIJIMA et al., 1980). The reason why the hindquarter flow was slightly but significantly increased after adrenalectomy is still obscure. Since the increase in hindquarter flow in the transposition response in adrenalectomized rats was still observed after $\beta$-adrenoceptor blockade (Fig. 5, bottom), it cannot be ascribable to the $\beta$-vasodilator effect of catecholamines from nerve terminals. It is tempting to speculate that the sympathetic vasoconstrictor discharge to the hindquarters is inhibited in the transposition response, reciprocally to the discharge to the viscera (see below).

Since the decrease in mesenteric and renal flows in the transposition response was markedly diminished after adrenalectomy, it is certain that catecholamines from the adrenal medulla take part in limiting visceral flow in the response also. Thus it may be said that the catecholamines released from the adrenal medulla in the transposition response limit the blood flow to the viscera by the $\alpha$-effect and shift it to the skeletal muscles by the $\beta$-effect. Such a role of adrenaline as a blood flow redistributor was first assumed by Cannon (CANNON, 1914; CANNON and BRITTON, 1927) and advocated by Folkow et al. (1948). This study furnishes experimental evidence that such a mechanism does in fact operate in conscious animals.

Since the flow in the superior mesenteric and renal arteries was still decreased in the transposition response after adrenalectomy, although to less an extent than before, it is considered that vasoconstrictor discharge to the viscera is increased in the response, also contributing to the vasoconstriction in these areas. On the contrary, the decrease in flow in these areas during the grasp response was as marked after adrenalectomy as before. It may be said that the vasoconstrictor fiber discharge is increased non-specifically almost everywhere in the grasp response. In contrast to this the vasoconstrictor discharge pattern in the transposition response seems to be more specific, in such a way as to assist the blood-flow redistributing effect of adrenal adrenaline.

A possible explanation for the abatement of the decrease in superior mesenteric flow occurring during the transposition response after $\beta$-adrenoceptor blockade is that it was due to a concomitant increase of arterial pressure. The reason why this phenomenon was not observed for renal flow may be sought in 
the marked autoregulation in this vascular bed.

In a previous study we observed that cardiac output was still increased in the transposition response after adrenalectomy (IRIUCHIJIMA et al., 1980). Although the increase in hindquarter flow in the response was less marked after adrenalectomy, the decrease in superior mesenteric and renal flows in the response was significantly less. The carotid flow, which remained almost unchanged in the response before adrenalectomy, tended to increase after adrenalectomy. As a net result, cardiac output is considered to increase in the transposition response after adrenalectomy as well as before.

The present study suggests that the functional significance of the abundant distribution of $\beta$-adrenoceptors in the vascular bed of skeletal muscle is to increase blood flow to muscle in preparation for an anticipated increase in the requirement for nutritional supply, in response to catecholamines released from the adrenal medulla. This is similar to the significance assumed for the cholinergic sympathetic vasodilator fibers in carnivores (Folkow and NeIL, 1971). Since these kinds of nerve fibers are lacking in the rat (BOLME et al., 1970; IRIUCHIJIMA, 1979), it is quite understandable that the $\beta$-adrenergic vasodilator effect on circulating adrenaline is so marked in this species. Since $\beta$-receptor activity is also marked in man, contrary to the case with the dog (GREEN and KEPCHAR, 1959), a mechanism which is similar to that observed in the rat may also be present in man. In the squirrel monkey, SCHRAMm et al. (1971) reported the presence of a marked $\beta$-adrenergic vasodilator system, which is resistant to atropinization.

\section{REFERENCES}

Bolme, P., Novotny, J., Uvnäs, B., and Wright, P. G. (1970) Species distribution of sympathetic cholinergic vasodilator nerves in skeletal muscle. Acta Physiol. Scand., 78: 60-64.

Cannon, W. B. (1914) The emergency function of the adrenal medulla in pain and the major emotions. Am. J. Physiol., 33: 356-372.

Cannon, W. B. and Britton, S. W. (1927) Studies on the conditions of activity in endocrine glands. XX. The influence of motion and emotion on medulliadrenal secretion. Am. J. Physiol., 79: 433-465.

Folkow, B., Frost, J., and UVNÄs, B. (1948) Action of adrenaline, nor-adrenaline and some other sympathomimetic drugs on the muscular, cutaneous and splanchnic vessels of the cat. Acta Physiol. Scand., 15: 412-420.

Folkow, B. and NeIL, E. (1971) Circulation, Oxford Univ. Press, New York, pp. 344-349.

GreEN, H. D. and KePCHAR, J. H. (1959) Control of peripheral resistances in major systemic vascular beds. Physiol. Rev., 39: 617-686.

GreEn, R. D. and Miller, J. W. (1966) Catecholamine concentrations: Changes in plasma of rats during estrous cycle and pregnancy. Science, 151: 825-826.

IRIUCHIJMA, J. (1979) Evidence against the presence of sympathetic vasodilator fibers in the rat. In: Integrative Control Functions of the Brain, Vol. 2, ed. by ITO, M. Kodansha, Tokyo, pp. 239-240.

IrIUChIJIMA, J., Teranishi, Y., and EgUCHI, C. (1980) Transposition response, a cardiovascular response to change of habitat in the rat. Jpn. J. Physiol., 30: 887-896.

Schramm, L. P., Honig, C. R., and Bignall, K. E. (1971) Active muscle vasodilation in primates homologous with sympathetic vasodilation in carnivores. Am. J. Physiol., 221: 768-777.

SHEPHERD, D. M. and West, G. B. (1951) Noradrenaline and the suprarenal medulla. Br. J. Pharmacol., 6: 665-674. 